\title{
A DIFFERENTIALLY ALGEBRAIC ELIMINATION THEOREM WITH APPLICATION TO ANALOG COMPUTABILITY IN THE CALCULUS OF VARIATIONS
}

\author{
LEE A. RUBEL ${ }^{1}$ AND MICHAEL F. SINGER
}

\begin{abstract}
An elimination theorem is proved in differential algebra, from which it follows that an analytic solution of virtually any ordinary differential equation that you can "write down" must actually solve an algebraic differential equation. As a corollary, it follows that the solutions of a large class of variational problems can be produced by an analog computer.
\end{abstract}

A hanging chain hangs in a catenary-essentially $y=\cosh x$, which satisfies the algebraic differential equation $y^{\prime 2}-y^{2}+1=0$. It is similar for other classical problems in the calculus of variations in one independent variable. One could ask: Is this an accident? Why doesn't the chain hang as $y=\Gamma(x)$ or some other "bad" function, which by [HOL] satisfies no algebraic differential equation? In this paper we give the reason by proving a general theorem (see Theorem 2 ).

This theorem is a direct consequence of an elimination theorem for differential equations (see Theorem 1) which implies, for example, that an analytic solution of

$$
y^{\prime y^{\prime \prime}}+\left(x^{2}+1\right) \sin \left(\frac{e^{y}+\tan y^{\prime}}{y^{\prime \prime \prime}}\right)+J_{0}\left(x+y^{\prime \prime}\right)=-\log x
$$

(where $J_{0}$ is the Bessel function) must actually satisfy some algebraic differential equation (ADE), i.e. an equation of the form

$$
P\left(x, y, y^{\prime}, \ldots, y^{(n)}\right)=0,
$$

where $P$ is a polynomial in all its variables. This result answers, in much greater generality than stated, Problem 12 of [RUB]. In a certain sense then, for virtually every ordinary differential equation that you can "write down," its solutions satisfy an algebraic differential equation.

Now the Shannon-Pour-El Thesis (see [SHA and POE], but see also [LIR] for further remarks) says essentially that a function $y(x)$ is the output of a generalpurpose analog computer if and only if it is differentially algebraic (DA), which means that it satisfies some ADE. We shall call such a function "analog-computable." (We need no new abbreviation-“DA" will suffice.)

Received by the editors September 26, 1984.

1980 Mathematics Subject Classification. Primary 34A34.

${ }^{1}$ Supported by the National Science Foundation. 
When it comes to (smooth) functions $f$ of several variables $x_{1}, \ldots, x_{k}$, we say that $f \in \mathrm{DA}$ if it is DA as a function of each variable when the others are held fixed. Ostrowski has shown [OST] that $f\left(x_{1}, \ldots, x_{n}\right)$ is DA if and only if it satisfies an algebraic Mayer system of partial differential equations. This means that there are a certain number of the (mixed) partial derivatives of $f$, say $p_{1}, \ldots, p_{l}$, such that if $p$ is any (mixed) partial derivative of $f$, then there is a rational function $H_{p}$ such that

$$
p=H_{p}\left(x_{1}, \ldots, x_{n}: p_{1}, \ldots, p_{l}\right) \text {. }
$$

For example,

$$
\frac{\partial^{2} f}{\partial x^{2}}+\frac{\partial^{2} f}{\partial y^{2}}=0 \quad \frac{\partial^{2} f}{\partial x \partial y}=0
$$

is an algebraic Mayer system.

(It is also easy to show (see [OST]) that $f\left(x_{1}, \ldots, x_{n}\right)$ is a DA function of its variables $x_{1}, \ldots, x_{n}$ if and only if, for all choices $x_{1}(t), \ldots, x_{n}(t)$ of DA functions of one variable, $F(t)=f\left(x_{1}(t), \ldots, x_{n}(t)\right)$ is a DA function of one variable. Again, by [OST], it follows that sums, products, differences, quotients, compositions, inverses, derivatives, and integrals of DA functions are again DA. In other words, the usual processes of algebraic analysis keep one within the class of differentially algebraic functions.)

So another way to phrase our Theorem 2 is that if we have a problem in the calculus of variations in one dimension, and if the integrand of the integral to be minimized is analog-computable, then the solution is also analog-computable. Whether this carries over to higher dimensions (where the constraints are also supposed to be analog-computable) is an open problem that seems difficult.

One ramification of this is the open problem, related to the Dirichlet problem for Laplace's equation in the disc.

Problem. Suppose $a_{n}$ are real (or complex) numbers with $\left|a_{n}\right| \leqslant 1 / n$ ! for $n=$ $0,1,2,3, \ldots$ and suppose that $\sum a_{n} \cos n x$ satisfies an algebraic differential equation. Must then $\sum a_{n} \sin n x$ satisfy a (presumably different) algebraic differential equation?

A second consequence of our elimination theorem is a differentially algebraic analog of the theorem of Weierstrass which considers meromorphic functions $W$ that satisfy an addition formula of the form

$$
W(u+v)=A(W(u), W(v)),
$$

where $A$ is an algebraic function of two variables. He proved that $W$ must be either (a) an algebraic function, (b) an algebraic function of some $e^{\lambda u}$, or (c) an algebraic function of some elliptic function. (See [ASK, Lecture 4] for this and related material.) We prove, in Theorem 3, that a meromorphic function $W$ satisfies a formula (*) where $A$ is differentially algebraic if and only if $W$ is differentially algebraic. It is challenging to ask what happens when this restriction on $A$ is strengthened to "linearly differentially algebraic," which means that, for every fixed $t, A(s, t)$ satisfies, in $s$, a linear differential equation with polynomial coefficients, with a similar condition on $A(s, t)$ as a function of $t$ for fixed $s$.

With these preliminaries done, we may begin the body of the paper. 
THEOREM 1. Let $G\left(t_{-1}, t_{0}, t_{1}, \ldots, t_{n}\right)$ be a nontrivial analytic differentially algebraic function of its $n+2$ variables, and let $y(x)$ be an analytic solution, say, on an interval $I$, of the differential equation

$$
G\left(x, y(x), y^{\prime}(x), \ldots, y^{(n)}(x)\right)=0 .
$$

Then $y(x)$ satisfies some algebraic differential equation on $I$.

Proof. For simplicity, we will handle the first-order case where (1) reads

$$
G\left(x, y(x), y^{\prime}(x)\right)=0 .
$$

The general case goes the same way, but with more complicated notation.

We may suppose that there is a subinterval $I^{\prime}$ of $I$ throughout which the separant, $S=\partial G / \partial y^{\prime}$ evaluated along our solution $y(x)$, does not vanish: $\partial G / \partial y^{\prime} \neq 0$.

(For if $\partial G / \partial y^{\prime}=0$ in an interval, then we could replace $G$ by $\partial G / \partial y^{\prime}$. We then have to worry about $\partial^{2} G / \partial\left(y^{\prime}\right)^{2}=0$ in a subinterval. Proceeding stepwise in this fashion, we arrive at an expression whose separant does not vanish, unless there is a point where $\partial^{n} G / \partial\left(y^{\prime}\right)^{n}=0$ for all $n$. But then, by analyticity, $G$ is independent of $y^{\prime}$ (the highest-order derivative) and we are reduced to the case of a lower-order differential equation. So an argument by induction on the order completes the proof.)

Let us write

$$
\begin{gathered}
G\left(x, y(x), y^{\prime}(x)\right)=0, \\
\frac{d}{d x} G\left(x, y(x), y^{\prime}(x)\right)=0 .
\end{gathered}
$$

By the introductory remarks about algebraic Mayer systems, there are finitely many (mixed) partial derivatives $\varphi_{1}, \ldots, \varphi_{k}$ of $G$ on which all the (mixed) partial derivatives of $G$ rationally depend.

Now in $\left(A_{1}\right)$, perform the indicated differentiation by the chain rule, to get

$$
\left(\mathrm{A}_{1}^{\prime}\right) \quad \frac{\partial G}{\partial x}+\frac{\partial G}{\partial y} y^{\prime}+S y^{\prime \prime}=0,
$$

and solve for $y^{\prime \prime}$ to get

$$
y^{\prime \prime}=-\frac{\partial G / \partial x+(\partial G / \partial y) y^{\prime}}{S} .
$$

Now differentiate formula $\left(\mathrm{B}_{2}\right)$ successively to get formulas $\left(\mathrm{B}_{3}\right),\left(\mathrm{B}_{4}\right), \ldots,\left(\mathrm{B}_{N+1}\right)$ for $y^{(3)}, y^{(4)}, \ldots, y^{(N+1)}$ as just polynomials in $y^{\prime}$ and various partial derivatives of $G$, all divided by powers of $S$. This is because at each stage $\left(\mathrm{B}_{3}\right),\left(\mathrm{B}_{4}\right), \ldots,\left(\mathrm{B}_{N+1}\right)$, successively, you may replace the intruding $y^{\prime \prime}$ by the expression for it in $\left(\mathrm{B}_{2}\right)$. Further, express all the partial derivatives of $G$ (including $S$ ) that occur, as rational functions of $\varphi_{1}, \varphi_{2}, \ldots, \varphi_{k}$. We arrive at

$$
\begin{aligned}
y^{\prime \prime}(x) & =R_{2}\left(x, y(x), y^{\prime}(x), \varphi_{1}, \ldots, \varphi_{k}\right), \\
y^{\prime \prime \prime}(x) & =R_{3}\left(x, y(x), y^{\prime}(x), \varphi_{1}, \ldots, \varphi_{k}\right), \\
& \vdots \\
y^{(N+1)}(x) & =R_{N+1}\left(x, y(x), y^{\prime}(x), \varphi_{1}, \ldots, \varphi_{k}\right) .
\end{aligned}
$$


Here, $\varphi_{i}=\varphi_{i}\left(x, y(x), y^{\prime}(x)\right)$. Hence, if $N$ is large enough, $N \geqslant(k+3)$, we will have so many rational functions of so few $(k+3)$ variables that there will be a nonzero polynomial $P$ such that $P\left(R_{1}, \ldots, R_{n}\right) \equiv 0$. We then have

$$
P\left(y^{\prime \prime}(x), y^{\prime \prime \prime}(x), \ldots, y^{(N+1)}(x)\right)=0
$$

on $I^{\prime}$, and hence on $I$ by analytic continuation. Thus, $y(x)$ satisfies the ADE (3), and the theorem is proved.

THEOREM 2. Suppose that the function $\bar{y}(x)$, analytic on $[a, b]$, minimizes

$$
\int_{a}^{b} F\left(x, y(x), y^{\prime}(x), \ldots, y^{(n)}(x)\right) d x
$$

subject, say, to

$$
\left\{\begin{array}{rlrl}
y(a) & =y_{0}^{*} & y(b) & =y_{0}^{\#} \\
y^{\prime}(a) & =y_{1}^{*} & y^{\prime}(b) & =y_{1}^{\#} \\
& \vdots & \vdots \\
y^{(n-1)}(a) & =y_{n-1}^{*} & y^{(n-1)}(b) & =y_{n-1}^{\#}
\end{array}\right.
$$

and that $F$ is a differentially algebraic analytic function of its $n+2$ variables on the range of $\left(x, \bar{y}(x), \ldots, \bar{y}^{(n)}\left(x_{-}\right)\right)$for $x \in[a, b]$. It is further assumed that $\int_{a}^{b} F d x$ does not depend only on the boundary conditions $(\mathrm{C})$. Then $\bar{y}(x)$ must be differentially algebraic.

Proof. For simplicity, we will again restrict ourselves to the case $n=1$; the general case goes similarly. Now $\bar{y}(x)$ must solve Euler's differential equation (see [COH, Vol. 1, p. 185])

$$
y^{\prime \prime} F_{y^{\prime} y^{\prime}}+y^{\prime} F_{y^{\prime} y}+F_{y^{\prime} x}-F_{y}=0 .
$$

But (see our introductory remarks about operations that preserve the class DA), the left-hand side of (4) is a DA function of its variables $x, y, y^{\prime}, y^{\prime \prime}$. By the additional assumption on the dependence of the integral, we see by $\$ 5$ of Chapter IV of Vol. 1 of $[\mathbf{C O H}]$ that the differential equation (4) is not the trivial equation $0=0$. By Theorem 1 , then, $\bar{y}(x)$ must be differentially algebraic, and the theorem is proved.

The following example shows that the analyticity assumption on $y(x)$ in Theorem 1 cannot be relaxed to mere $C^{n}$.

ExAmple. Consider the functions defined by $e_{0}(x)=x, e_{n}(x)=\exp \left(e_{n-1}(x)\right)$. For each $n, e_{n}(x)$ and all its derivatives are positive on $\mathbf{R}$. It is easy to show (see [BAB] for a more general result) that $e_{n+1}(x)$ satisfies no ADE of order $n$. Fix $n$, and let $y(x)=e_{n+1}(x)$ for $x \geqslant 0$, and

$$
y(x)=p(x)=e_{n+1}(0)+e_{n+1}^{\prime}(0) x+\cdots+e_{n+1}^{(n)}(0)\left(x^{n} / n !\right)
$$

for $x<0$. Notice that $y(x) \in C^{n}(\mathbf{R})$ but that $y(x) \notin C^{n+1}(\mathbf{R})$. Now $y(x)$ satisfies the differential equation

$$
(y-p(x))\left(y-e_{n+1}(x)\right)=0
$$


on all of $\mathbf{R}$. However, $y(x)$ satisfies no ADE on all of $\mathbf{R}$, since the ADE would have to be of order at most $n$, since $y^{(n+1)}(0)$ fails to exist. This would mean that $e_{n+1}(x)$ would satisfy an $n$th order ADE on $\mathbf{R}$, which is a contradiction. We do not know about the $C^{\infty}$ case.

The problem of whether the analog of Theorem 2 holds in more than one variable seems to involve new difficulties. For example, let $\Omega$ be a Jordan region in $\mathbf{R}^{2}$, bounded by a differentially algebraic curve $\gamma$; say $\Omega$ is the unit disc and $\gamma$ the unit circle. Suppose we have a differentially algebraic function $u_{0}$ on $\gamma$, and consider the problem of minimizing

$$
\iint_{\Omega}\left[\left(\frac{\partial u}{\partial x}\right)^{2}+\left(\frac{\partial u}{\partial y}\right)^{2}\right] d x d y
$$

subject to $\left.u\right|_{\gamma}=u_{0}$.

Must the solution $\bar{u}(x, y)$ be differentially algebraic? Put somewhat picturesquely, we are asking whether heat flow is an analog computer. A related problem, involving the Hilbert transform, is the following: Some music is being played, in the next room, by an analog orchestra, but you hear the music through the wall, which filters out all the frequencies above a certain threshold $t_{0}$. Is the music you hear produced by an analog computer? In short, is a band-pass filter an analog device in the sense of [POE]?

THEOREM 3. In order that the meromorphic function $W$ should satisfy an addition formula

$$
W(u+v)=A(W(u), W(v)),
$$

where $A$ is a differentially algebraic analytic function of its two variables, it is necessary and sufficient that $W$ be a differentially algebraic function of its one variable.

Remark. A little care is needed in the hypothesis about the region where $W$ is defined, so that $W(u+v)$ should make sense. We leave the details of this to the reader. Further, other functional relations than $(*)$ can be treated in the same way, as long as they are differentially algebraic, like

$$
W(u+v+\sin (u v))=A(W(u), W(v)),
$$

but we stick to $(*)$ for convenience.

Proof of Theorem 3. First, suppose that $W \in$ DA. Then it satisfies (*) with

$$
A(s, t)=W\left(W^{-1}(s)+W^{-1}(t)\right),
$$

and $A$ is DA because it is separately so. For the converse, take derivatives of (*) first with respect to $u$, and then with respect to $v$, to get

$$
\begin{aligned}
W^{\prime}(u+v) & =A_{1}(W(u), W(v)) W^{\prime}(u) \\
& =A_{2}(W(u), W(v)) W^{\prime}(v),
\end{aligned}
$$

where $A_{1}(s, t)=\partial A(s, t) / \partial s, A_{2}(s, t)=\partial A(s, t) / \partial t$. Choose a value $v_{0}$ so that $A_{1}\left(W(u), W\left(v_{0}\right)\right)$ is not the zero function. (If this is impossible, then we have 
$\partial A(s, t) / \partial t \equiv 0$, so that $A(s, t)$ would be a function of $s$ alone, so that (*) would read $W(u+v)=A(W(u))$, so that $W$ must be a constant, which is certainly DA.) Then look at the differential equation

(\#) $\quad A_{1}\left(W(u), W\left(v_{0}\right)\right) W^{\prime}(u)-A_{2}\left(W(u), W\left(v_{0}\right)\right) W^{\prime}\left(v_{0}\right)=0$.

This equation is of the type treated in Theorem 1 , and we conclude from that theorem that $W \in \mathrm{DA}$. This ends the paper.

\section{REFERENCES}

[ASK] Richard Askey, Orthogonal polynomials and special functions, SIAM, Philadelphia, Pa., 1975.

[BAB] Ararat Babakhanian, Exponentials in differentially algebraic extension fields, Duke Math. J. 40 (1973), 455-460.

[COH] Richard Courant and David Hilbert, Methods of mathematical physics, Interscience, New York, 1966.

[HOL] O. Hölder, Ueber die Eigenschaft der Gamma Funktion keineralgebraischen Differentialgleichung zu genügen, Math. Ann. 28 (1887), 1-13.

[LIR] Leonard Lipshitz and Lee A. Rubel, $A$ differentially algebraic replacement theorem, and analog computability, Preprint, Fall 1984.

[OST] Alexander Ostrowski, Uber Dirichletsche Reihen und algebraische Differentialgleichungen, Math. Z. 8 (1920), 241-298.

[POE] Marian Boykan Pour-El, Abstract computability and its relation to the general-purpose analog computer, etc., Trans. Amer. Math. Soc. 199 (1974), 1-29.

[RUB] Lee A. Rubel, Some research problems about algebraic differential equations, Trans. Amer. Math. Soc. 280 (1983), 43-52.

[SHA] Claude Shannon, Mathematical theory of the differential analyzer, J. Math. Phys. 20 (1941), 337-354.

Department of Mathematics, University of Illinois at Urbana - Champaign, 1409 West Green STREeT, URBANA, Illinois 61801

Department of Mathematics, North Carolina State University, Raleigh, North Carolina 27650 